Research Article

\title{
A study of role of diagnostic and therapeutic laparoscopy in chronic and recurrent abdominal pain
}

\author{
Rajeev Karvande, Ranjeet Kamble*, Manoj Kharade
}

Department of Surgery, LTMMC and LTMGH, Sion, Mumbai, Maharashtra, India

\author{
Received: 01 July 2016 \\ Accepted: 15 July 2016 \\ *Correspondence: \\ Dr. Ranjeet Kamble, \\ E-mail: drranjeetkamble@yahoo.co.in
}

Copyright: () the author(s), publisher and licensee Medip Academy. This is an open-access article distributed under the terms of the Creative Commons Attribution Non-Commercial License, which permits unrestricted non-commercial use, distribution, and reproduction in any medium, provided the original work is properly cited.

\begin{abstract}
Background: Chronic abdominal pain is a common complaint of the patients seeking a primary care physician. It leads to evident suffering and disability, both physically and psychologically and it is associated with poor quality of life. Many diagnostic and therapeutic procedures have been described in literature, in that laparoscopy is one of the modalities that could be of benefit in such cases. Hence, the aim of the study was to evaluate the role of diagnostic and therapeutic laparoscopy in chronic and recurrent idiopathic abdominal and also to assess the epidemiology, clinical features, diagnostic modalities and management of chronic and recurrent abdominal pain.

Methods: This is a single centre, prospective study done on 63 patients with chronic abdomen pain. The pain in all patients was of unknown etiology despite all the investigative procedures. All patients were subjected to laparoscopic evaluation for their conditions. The findings and outcomes of the laparoscopy were recorded and analyzed.

Results: In the present study Out of 63,26 were male 37 were female showing female preponderance $(58.7 \%)$ of patients presenting with chronic and recurrent abdominal pain with mean age of presentation 31.7 years. The majority of the patients in our study presented with pain in right lower quadrant pain $(n=43)$ followed by pain in periumbilical region $(n=7)$. Others had pain in other quadrants of abdomen. Only 6 patients had diffuse abdominal pain. In our study of 63 patients, the most common finding was chronic appendicular pathology, which was present in $56.1 \%$ patients, inflammatory or congenital band of adhesions noted in around $21.2 \%$ patients, abdominal Koch's was noted in $19.7 \%$, mesenteric lymphadenopathy noted in around $18.2 \%$, ovarian cyst findings were noted in around $7.6 \%$ patients, 2 patients had Meckel's diverticulum and 4 had normal findings. $92.08 \%(n=58)$ patients had positive response (Reduction or complete relief of pain) at the end of one month in relation to effectiveness of diagnostic laparoscopy combined with a therapeutic procedure while $88.88 \%(n=56)$ had positive response at the end of two months.

Conclusions: Laparoscopy is a safe, quick and effective modality of investigation for chronic abdominal pain. Not only does laparoscopy point to a diagnosis, it has the added advantage that therapeutic intervention can be done at the same sitting in most cases thus avoiding another hospitalization or another exploration of the abdomen. Diagnostic laparoscopy has a high diagnostic and therapeutic efficacy.
\end{abstract}

Keywords: Diagnostic, Therapeutic laparoscopy, Recurrent abdominal pain, Epidemiology

\section{INTRODUCTION}

Population based studies have shown that chronic abdominal pain is a pervasive problem. Abdominal pain was the third most common pain complaint of individuals enrolled in a large health maintenance organization.
Chronic abdominal pain is defined as recurrent abdominal pain on and off for more than three months duration. It leads to evident suffering and disability in patient both physically and psychologically. Chronic abdominal pain is associated with poor quality of life. ${ }^{1}$ 
Patients with chronic abdominal pain undergo numerous diagnostic studies, but their pain remains an undiagnosed entity even after numerous investigations. ${ }^{2-5}$ More than $40 \%$ of the patients have no diagnosis after extensive workup and hence is often referred as unexplained chronic abdominal pain (UCAP). Sometimes abdominal wall pain is also common and frequently mistaken for visceral pain. Hence, diagnostic laparoscopy is an important option to explore a patient's abdomen and performing laparotomy. ${ }^{6}$

With advances in optics, laparoscopy allows visualization of entire peritoneal cavity and further makes possible histological diagnosis of target biopsy under vision. The rapid recovery and return to normal activity that follows laparoscopic surgery is an added incentive for the surgeons to adopt more laparoscopic techniques. Hence, the present study is aimed to evaluate the role of diagnostic and therapeutic laparoscopy in chronic and recurrent idiopathic abdominal and also to assess the epidemiology, clinical features, diagnostic modalities and management of chronic and recurrent abdominal pain.

\section{METHODS}

A single centre, prospective study done at a tertiary care hospital in a metropolitan city, LTMG hospital, Mumbai over period of 2 years from $1^{\text {st }}$ Dec 2013 to $30^{\text {th }}$ Nov 2015. 63 patients were included in study following inclusion criteria.

\section{Inclusion criteria}

- All cases of undiagnosed (by conventional methods and investigations such as detailed history, clinical examination, blood counts, urine examination, USG abdomen, plain x-ray abdomen) chronic abdominal pain for more than three months duration in patients of more than 18 years of age.

- Cases of clinically diagnosed chronic abdominal pain of more than three months duration not responding to the treatment given.

\section{Exclusion criteria}

- Patients with identifiable process that can explain the cause of pain.

- Acute or inflammatory process.

- Patients with acute intestinal obstruction.

- Patients with coagulation defects, patients with critical illness, severe/decompensated cardiopulmonary failure and medically unfit for surgery.

- Age less than 18years and pregnant women.

- Large ventral hernia, diaphragmatic hernia.

- Bacterial peritonitis, abdominal wall infections.

The written informed consent was taken from all the subjects prior to all the procedures. A detailed history was taken from each of the patient as per the proforma designed before the commencement of the study. The clinical examination findings were also recorded in the proforma.

The recorded data included particulars of the patient, duration of illness, site of abdominal pain, past history of surgical explorations, co-morbid conditions, investigations. Subsequently the intra operative findings, therapeutic/ diagnostic intervention done, correlation of the intra operative findings with the histopathology report, complications during the intra and post-operative period and the relief from the pain were recorded and analysed. The routine investigations like blood and urine analysis, ECG, ultra sonogram and chest X-ray were done. And in cases when required the following investigations were also done like ESR, fasting blood sugar and post prandial blood sugar and imaging like erect X-ray abdomen, barium studies, esophago gastroduodenoscopy, colonoscopy, computerized tomography of the abdomen.

All surgeries were carried out under general anesthesia. All patients had a Ryle's tube inserted and bladder catheterized prior to anesthesia. Pneumoperitoneum was created using Hasson's technique. A 10mm umbilical camera port was inserted and two lateral $5 \mathrm{~mm}$ ports depending on the organ of interest and the suspected pathology. The sites of port insertion varied depending on the presence or absence of previous abdominal surgery scars. Diagnostic laparoscopy of the abdomen was carried out carefully inspecting the entire visceral contents of the abdomen for any pathology. Starting from the liver, the gall bladder, anterior surface of the stomach, large intestine, entire length of small intestine with particular emphasis on appendix and terminal ileum, anterior surfaces of the retroperitoneal organs, uterus, fallopian tubes and ovaries and peritoneal surface. An adhesion between the bowel loops or to the anterior abdominal wall was also looked for. The surgical procedure carried out was depending on the intra operative findings and as per indications which ranged from biopsy from suspicious lesions to adhesiolysis to appendectomy. All the ports were closed using absorbable suture materials at the end of the procedure.

\section{RESULTS}

The total number of patients included in the study was 63 after meeting the inclusion criteria. Out of 63 cases 37 were female and 26 were male showing female preponderance $(58.7 \%)$ of patients presenting with chronic and recurrent abdominal pain. Our study showed a peak incidence of chronic pain in abdomen in the third decade as observed in Table 1 . The youngest patient was 18 years and the oldest patient being 65 years old. The mean age of presentation was 31.7 years.

In our study around $73 \%(n=46)$ of the patients reported pain in abdomen of duration between 3 to 12 months. 11 
patients $(17.46 \%)$ had abdominal pain between 12 to 18 months, 4 patients $(6.34 \%)$ had pain from 18 to 36 months while only 2 patients $(3.17 \%)$ had pain for more than 36 months. The majority of the patients in our study presented with pain in right lower quadrant pain $(n=43)$ followed by pain in peri-umbilical region $(n=7)$. Others had pain in other quadrants of abdomen. Only 6 patients had diffuse abdominal pain.

\section{Table 1: Age distribution of patients presenting with chronic abdominal pain.}

\begin{tabular}{|lll|}
\hline Age group (in years) & Frequency & Percentage \\
\hline $18-30$ & 33 & 52.4 \\
\hline $31-40$ & 18 & 28.6 \\
\hline $41-50$ & 8 & 12.7 \\
\hline $51-60$ & 3 & 4.8 \\
\hline above 60 & 1 & 1.6 \\
\hline Total & 63 & 100.0 \\
\hline
\end{tabular}

In this study for all the 63 patients diagnostic lab findings were done. The distribution of findings at laparoscopy is as shown in Figure 1.

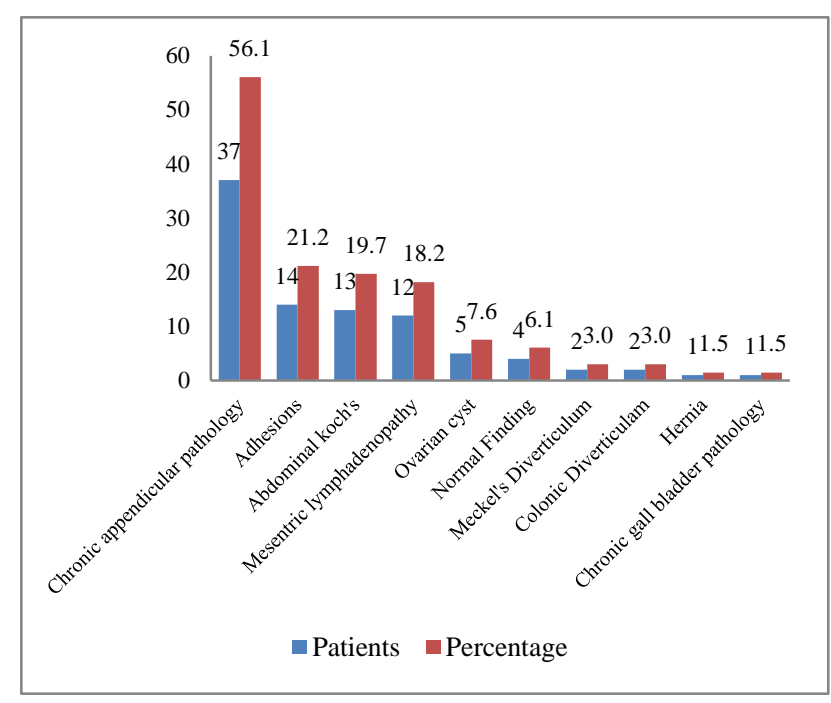

Figure 1: The distribution of findings at laparoscopy.

Table 2: Number of patients with previous history of abdominal surgery.

\begin{tabular}{|ll|}
\hline Type of surgery & No of patients \\
\hline Tubal ligation & 15 \\
\hline Appendectomy & 4 \\
\hline Hysterectomy & 3 \\
\hline LSCS & 2 \\
\hline Laparoscopic cholecystectomy & 1 \\
\hline Open cholecystectomy & 1 \\
\hline Laparotomy & 1 \\
\hline
\end{tabular}

The most common finding was chronic appendicular pathology, which was present in $56.1 \%$ patients. Ultra sonogram done preoperatively in all these patients showed normal finding. But on diagnostic laparoscopy appendix was seen as enlarged, thickened, curved, and wrapped with omentum or adherent to adjacent structures and histopathological examination confirmed the findings in almost all the cases.

The next most common finding observed was postoperative, inflammatory or congenital band of adhesions noted in around $21.2 \%$ patients. Almost all of them underwent laparoscopic adhesiolysis. In 14 patients with adhesions 9 had history of abdominal surgery in past $(64.28 \%)$.

The third most common finding was abdominal Koch's, which was noted in around $19.7 \%$ patient, which was shown by the presence of strictures in bowel, tubercles over bowel surface, omental caking, free fluid in abdomen, mesenteric lymphadenopathy. Endoscopic biopsy of peritoneum or omentum and free fluid analysis confirmed the intraoperative findings in all the patients. Patients were given AKT as per DOTS and resectionanastomosis was done for non-passable strictures.

Mesenteric lymphadenopathy was also noted in around $18.2 \%$ patients, which showed reactive lymphoid hyperplasia or non- specific lymphadenitis on biopsy in most of the cases. Mesenteric lymphadenitis was common in patients with recurrent upper respiratory tract infections or infectious disease of bowel like colitis, gastroenteritis or enteric fever. All of them were given a course of antibiotics with analgesics and were observed.

Ovarian cyst findings were noted in around $7.6 \%$ patients. Ovarian cyst aspiration was done in almost all of them and fluid was sent for analysis and patient was advised to take gynecologist opinion. Two patients had Meckel's diverticulum as the finding, of which one underwent resection and anastomosis and other was observed as it was broad based.

Four patients had normal findings. One patient had chronic gall bladder pathology with adhesions for which cholecystectomy was done along with adhesiolysis. Histopathological reports confirmed the findings and one patient had ascites, omental caking along with deposits in liver biopsy showed it as the adenocarcinoma of unknown primary with peritoneal metastasis.

After laboratory findings diagnostic laparoscopy was done in all the patients and it was noted that out of total 63 patients $37(56.1 \%)$ needed appendectomy, 11 (16.7\%) underwent adhesiolysis, 2 (3\%) underwent resection and anastomosis, $2(3 \%)$ required ovarian cyst aspiration while one $(1.5 \%)$ underwent cholecystectomy.

Previous history of abdominal surgery was also collected in the present study and it was found that around 25(40\%) of the patients in our study had undergone a previous abdominal surgery. The details were given in Table 2 . 
At the end of one month in relation to effectiveness of diagnostic laparoscopy combined with a therapeutic procedure $92.08 \%(n=58)$ patients had positive response (reduction or complete relief of pain) while $88.88 \%$ $(n=56)$ had positive response at the end of two months.

\section{DISCUSSION}

In our study there were 37 females and 26 male patients. The age group of the patients ranged from 18 years to 65 years with the average age being 31.7 years and the duration of pain ranged between 3 months to 5 years. From the results obtained from our study it is evident that females are more suffered with chronic abdomen pain and this statement is supported by the findings of Klingensmith et al, Thanaponsathron et al, Raymond et al and by EI-Labban GM et al..$^{7-10}$

The main cause of chronic abdominal pain in the present study was found to be appendicitis. In our study $56.1 \%$ patients had chronic appendicular pathology causing chronic abdominal pain in right lower quadrant (64\%). The cause and symptoms of the present study were similar as observed in studies of Baria, Fayez et al, Schrenk et al. ${ }^{11-13}$

In the study, diagnostic laparoscopy was done in 63 patient and the etiology was identified in around $93.65 \%$ (59) cases and these findings are comparable to previously done aforementioned studies of Andreollo et al and Salky et al. ${ }^{14,15}$ According to the studies of Andreollo the etiology identified by laproscopy was around $86.3 \%$ cases of 168 patients and in the study of Salky the percentage of identified cases was $76 \%$ of 265 patients.

In the present study, therapeutic efficacy was denoted by the percentage of patients who reported positive outcome (no pain or decrease in pain) at the time of follow up after 2 months. Positive outcome was noted in around $89 \%$ (56) cases and the therapeutic efficacy with diagnostic laparoscopy combined with a therapeutic procedure was comparable with previous studies conducted by Shayani et al. According to him the percentage of therapeutic efficacy in appendicitis was $77.8 \%$ out of 18 patients. The same similar finding was also observed with Chao $\mathrm{K}$ et al i.e., $78 \%$ out of 41 patients. ${ }^{16,17}$

In this study, of all the 37 patients who underwent laparoscopic appendectomy for appendicular pathology around $97.29 \%$ had positive response of complete relief or reduction in abdominal pain and this outcome is comparable with the previous studies done by Fayez et al. ${ }^{18}$ In his study patients with chronic abdominal pain undergoing appendectomy were reviewed and found that about $95 \%$ had resolution of pain and in a study performed by Raymond $\mathrm{P}$ et al, $74 \%$ patient reported resolution of pain. ${ }^{9}$

\section{CONCLUSION}

Laparoscopy has an effective diagnostic accuracy and therapeutic efficacy in the management of patients, who present to us with chronic abdominal pain, especially in whom conventional methods of investigations have failed to elicit a cause for the pain. It is a safe, quick and effective modality of investigation for chronic abdominal pain. Not only does laparoscopy point to a diagnosis, it has the added advantage that therapeutic intervention can be done at the same sitting in most cases thus avoiding another hospitalization or another exploration of the abdomen. Diagnostic laparoscopy has a high diagnostic and therapeutic efficacy. It prevents unnecessary laparotomy in a significant number of cases.

Funding: No funding sources Conflict of interest: None declared

Ethical approval: The study was approved by the institutional ethics committee

\section{REFERENCES}

1. American Acaedemy of Paediatrics Subcommittee on Chronic Abdominal Pain. Chronic Abdominal Pain in children. Pediatrics. 2005;115:812-5.

2. Camilleri M. Management of patients with chronic abdominal pain in clinical practice. Neurogastroenterol Motil. 2006;18:499-506.

3. Paajanen H, Julkunen K, Waris H. Laparoscopy in Chronic Abdominal Pain: A Prospective Nonrandomized Long-term Follow-up Study. J Clin Gastroenterol. 2005;39(2):110-4.

4. Townsend CO, Sletten CD, Bruce BK, Rome JD, Luedtke CA, Hodgson JE. Physical and emotional functioning of adult patients with chronic abdominal pain: Comparison with patients with chronic back pain. J Pain. 2005;6:5-83.

5. Mc Garrity TJ, Peters DJ, Thompson C, McGarrity SJ. Outcome of patients with chronic abdominal pain referred to chronic pain clinic. Am J Gastroenterol. 2000;95:1812-6.

6. Palanivelu C. Diagnostic laparoscopy- Indication, tuberculosis and adhesiolysis, In: Art of laparoscopic surgery, Textbook and atlas. 1st edition, vol 1, Jaypee publishers; 2005:152-177.

7. Klingensmith ME, Soybel DI, Brooks DC. Laparoscopy for chronic abdominal Pain. Surg Endosc. 1996;10(11):1085-7.

8. Thanapongsathron W, Kanjanabut B, Vaniyapong T, Thaworncharoen S. Chronic right lower quadrant abdominal pain: laparoscopic approach. J Med Assoc Thai. 2005;88:42-7.

9. Raymond P, Onders MD, Elizabeth A, Mittendorf MD. Utility of laparoscopy in chronic abdominal Pain. Surg. 2003;134(4):549-54.

10. El-labban GM, Hokkam EN. The efficacy of laparoscopy in the diagnosis and management of chronic abdominal pain. J minim Access Surg. 2010;6(4):95-9. 
11. Baria KAK. Role of laparoscopy in diagnosis and management of chronic Abdominal pain. Indian $\mathbf{J}$ Sci Res. 2013;4(1):65-8.

12. Karl Miller MD, Edith Mayer MD, Erich Moritz HD. The role of laparoscopy in chronic\& recurrent abdominal Pain. Am J Surg. 1996;172:353-7.

13. Schrenk P, Woisetschläger R, Wayand WU, Rieger R, Sulzbacher H. Diagnostic laparoscopy: a survey of 92 patients. Am J Surg. 1994;168(4):348-51.

14. Andreollo NA, Coelho Neto Jde S, Lopes LR, Brandalise NA, Leonardi LS. Laparoscopy in the diagnosis of intra-abdominal diseases. Analysis of 168 cases. Rev Assoc Med Bras. 1999;45(1):34-8.
15. Salky BA, Edye MB. The role of laparoscopy in the diagnosis and treatment of abdominal Pain syndromes. Surg Endosc. 1998;12(7):911-4.

16. Shayani V, Siegert C, Favia P. The Role of Laparoscopic Adhesiolysis in the Treatment of Patients with Chronic Abdominal Pain or Recurrent Bowel Obstruction. JSLS. 2002;6(2):111-4.

17. Chao K, Farrell S, Kerdemelidis P, Tulloh B. Diagnostic laparoscopy for chronic right iliac fossa pain: a pilot study. Aust N Z J Surg. 1997;67(11):789-91.

18. Fayez JA, Toy NJ, Flanagan TM. The appendix as the cause of chronic lower abdominal pain. Am J Obstet Gynecol. 1995;172:122-3.

Cite this article as: Karvande R, Kamble R, Kharade M. A study of role of diagnostic and therapeutic laparoscopy in chronic and recurrent abdominal pain. Int Surg J 2016;3:1336-40. 